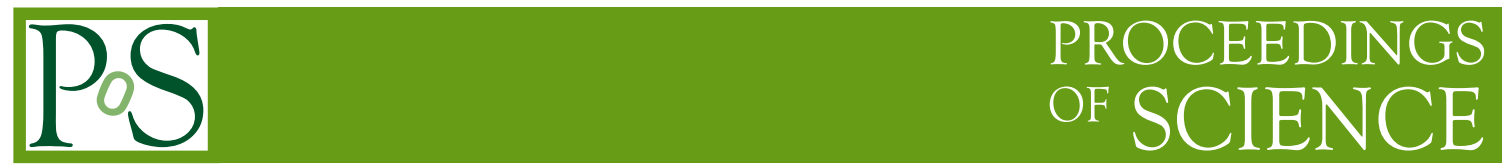

\title{
The commissioning of the CUORE experiment: the mini-tower run
}

\author{
S. Copello ${ }^{* 16,8}$, C. Alduino ${ }^{1}$, K. Alfonso ${ }^{2}$, F. T. Avignone $\mathrm{III}^{1}$, O. Azzolini ${ }^{3}$, G. Bari ${ }^{4}$, \\ F. Bellini ${ }^{5,6}$, G. Benato ${ }^{7}$, A. Bersani ${ }^{8}$, M. Biassoni ${ }^{9,10}$, A. Branca $^{11,12}$, C. Brofferio ${ }^{9,10}$, \\ C. Bucci ${ }^{13}$, A. Camacho ${ }^{3}$, A. Caminata ${ }^{8}$, L. Canonica ${ }^{14,13}$, X. G. Cao ${ }^{15}$, S. Capelli ${ }^{9,10}$, \\ L. Cappelli ${ }^{13}$, L. Cardani $^{6}$, P. Carniti ${ }^{9,10}$, N. Casali ${ }^{6}$, L. Cassina ${ }^{9,10}$, D. Chiesa ${ }^{9,10}$, \\ N. Chott ${ }^{1}$, M. Clemenza ${ }^{9,10}$, C. Cosmelli ${ }^{5,6}$, O. Cremonesi ${ }^{10}$, R. J. Creswick ${ }^{1}$, \\ J. S. Cushman ${ }^{17}$, A. D’Addabbo ${ }^{13}$, D. D’Aguanno ${ }^{13,18}$, I. Dafinei ${ }^{6}$, C. J. Davis ${ }^{17}$, \\ S. Dell'Oro ${ }^{19,13,20}$, M. M. Deninno ${ }^{4}$, S. Di Domizio ${ }^{16,8}$, M. L. Di Vacri ${ }^{13,21}$, \\ A. Drobizhev ${ }^{7,22}$, D. Q. Fang ${ }^{15}$, M. Faverzani ${ }^{9,10}$, E. Ferri ${ }^{10}$, F. Ferroni ${ }^{5,6}$, E. Fiorini ${ }^{10,9}$, \\ M. A. Franceschi ${ }^{23}$, S. J. Freedman ${ }^{22,7, a}$, B. K. Fujikawa ${ }^{22}$, A. Giachero ${ }^{10}$, \\ L. Gironi ${ }^{9,10}$, A. Giuliani ${ }^{24}$, L. Gladstone ${ }^{14}$, P. Gorla ${ }^{13}$, C. Gotti ${ }^{9,10}$, T. D. Gutierrez ${ }^{25}$, \\ K. Han $^{26}$, K. M. Heeger ${ }^{17}$, R. Hennings-Yeomans ${ }^{7,22}$, H. Z. Huang ${ }^{2}$, G. Keppel ${ }^{3}$, \\ Yu. G. Kolomensky ${ }^{7,22}$, A. Leder ${ }^{14}$, C. Ligi ${ }^{23}$, K. E. Lim ${ }^{17}$, Y. G. Ma ${ }^{15}$, L. Marini ${ }^{16,8}$, \\ M. Martinez ${ }^{5,6,27}$, R. H. Maruyama ${ }^{17}$, Y. Mei ${ }^{22}$, N. Moggi ${ }^{28,4}$, S. Morganti ${ }^{6}$, \\ S. S. Nagorny ${ }^{13,20}$, T. Napolitano ${ }^{23}$, M. Nastasi ${ }^{9}, 10$, C. Nones ${ }^{29}$, E. B. Norman ${ }^{30,31}$, \\ V. Novati ${ }^{24}$, A. Nucciotti ${ }^{9,10}$, I. Nutini ${ }^{13,20}$, T. O'Donnell ${ }^{19}$, J. L. Ouellet ${ }^{14}$, \\ C. E. Pagliarone ${ }^{13,18}$, M. Pallavicini ${ }^{16,8}$, V. Palmieri' ${ }^{3}$, L. Pattavina ${ }^{13}$, M. Pavan $^{9,10}$, \\ G. Pessina ${ }^{10}$, C. Pira ${ }^{3}$, S. Pirro ${ }^{13}$, S. Pozzi ${ }^{9}{ }^{10}$, E. Previtali ${ }^{10}$, F. Reindl ${ }^{6}$, C. Rosenfeld ${ }^{1}$, \\ C. Rusconi ${ }^{1,13}$, M. Sakai ${ }^{2}$, S. Sangiorgio ${ }^{30}$, D. Santone ${ }^{13,21}$, B. Schmidt ${ }^{22}$, \\ J. Schmidt ${ }^{2}$, N. D. Scielzo ${ }^{30}$, V. Singh ${ }^{7}$, M. Sisti ${ }^{9,10}$, L. Taffarello ${ }^{11}$, F. Terranova ${ }^{9,10}$, \\ C. Tomei $^{6}$, M. Vignati ${ }^{6}$, S. L. Wagaarachchi ${ }^{7,22}$, B. S. Wang ${ }^{30,31}$, H. W. Wang ${ }^{15}$, \\ B. Welliver ${ }^{22}$, J. Wilson ${ }^{1}$, K. Wilson ${ }^{1}$, L. A. Winslow ${ }^{14}$, T. Wise ${ }^{17,32}$, L. Zanotti ${ }^{9,10}$, \\ G. Q. Zhang ${ }^{15}$, S. Zimmermann ${ }^{33}$, and S. Zucchelli ${ }^{28,4}$
}


${ }^{1}$ Department of Physics and Astronomy, University of South Carolina, Columbia, SC 29208, USA

${ }^{2}$ Department of Physics and Astronomy, University of California, Los Angeles, CA 90095, USA

${ }^{3}$ INFN - Laboratori Nazionali di Legnaro, Legnaro (Padova) I-35020, Italy

${ }^{4}$ INFN - Sezione di Bologna, Bologna I-40127, Italy

5 Dipartimento di Fisica, Sapienza Università di Roma, Roma I-00185, Italy

${ }^{6}$ INFN - Sezione di Roma, Roma I-00185, Italy

${ }^{7}$ Department of Physics, University of California, Berkeley, CA 94720, USA

${ }^{8}$ INFN - Sezione di Genova, Genova I-16146, Italy

${ }^{9}$ Dipartimento di Fisica, Università di Milano-Bicocca, Milano I-20126, Italy

${ }^{10}$ INFN - Sezione di Milano Bicocca, Milano I-20126, Italy

11 INFN - Sezione di Padova, Padova I-35131, Italy

12 Dipartimento di Fisica e Astronomia, Università di Padova, I-35131 Padova, Italy

${ }^{13}$ INFN - Laboratori Nazionali del Gran Sasso, Assergi (L'Aquila) I-67100, Italy

${ }^{14}$ Massachusetts Institute of Technology, Cambridge, MA 02139, USA

15 Shanghai Institute of Applied Physics, Chinese Academy of Sciences, Shanghai 201800, China

${ }^{16}$ Dipartimento di Fisica, Università di Genova, Genova I-16146, Italy

17 Department of Physics, Yale University, New Haven, CT 06520, USA

${ }^{18}$ Dipartimento di Ingegneria Civile e Meccanica, Università degli Studi di Cassino e del Lazio Meridionale, Cassino I-03043, Italy

${ }^{19}$ Center for Neutrino Physics, Virginia Polytechnic Institute and State University, Blacksburg, Virginia 24061, USA

${ }^{20}$ INFN - Gran Sasso Science Institute, L'Aquila I-67100, Italy

${ }^{21}$ Dipartimento di Scienze Fisiche e Chimiche, Università dell'Aquila, L'Aquila I-67100, Italy

${ }^{22}$ Nuclear Science Division, Lawrence Berkeley National Laboratory, Berkeley, CA 94720, USA

${ }^{23}$ INFN - Laboratori Nazionali di Frascati, Frascati (Roma) I-00044, Italy

${ }^{24}$ CSNSM, Univ. Paris-Sud, CNRS/IN2P3, UniversitÃl' Paris-Saclay, 91405 Orsay, France

25 Physics Department, California Polytechnic State University, San Luis Obispo, CA 93407, USA

${ }^{26}$ INPAC and School of Physics and Astronomy, Shanghai Jiao Tong University; Shanghai Laboratory for Particle Physics and Cosmology, Shanghai 200240, China

27 Laboratorio de Fisica Nuclear y Astroparticulas, Universidad de Zaragoza, Zaragoza 50009, Spain

${ }^{28}$ Dipartimento di Fisica e Astronomia, Alma Mater Studiorum - Università di Bologna, Bologna I-40127, Italy

${ }^{29}$ Service de Physique des Particules, CEA / Saclay, 91191 Gif-sur-Yvette, France

${ }^{30}$ Lawrence Livermore National Laboratory, Livermore, CA 94550, USA

${ }^{31}$ Department of Nuclear Engineering, University of California, Berkeley, CA 94720, USA

32 Department of Physics, University of Wisconsin, Madison, WI 53706, USA

${ }^{33}$ Engineering Division, Lawrence Berkeley National Laboratory, Berkeley, CA 94720, USA

E-mail: scopello@ge.infn.it 
CUORE is a ton-scale experiment approaching the data taking phase in Gran Sasso National Laboratory. Its primary goal is to search for the neutrinoless double-beta decay in ${ }^{130} \mathrm{Te}$ using 988 crystals of tellurim dioxide. The crystals are operated as bolometers at about $10 \mathrm{mK}$ taking advantage of one of the largest dilution cryostat ever built. Concluded in March 2016, the cryostat commissioning consisted in a sequence of cool down runs each one integrating new parts of the apparatus. The last run was performed with the fully configured cryostat and the thermal load at $4 \mathrm{~K}$ reached the impressive mass of about 14 tons. During that run the base temperature of 6.3 $\mathrm{mK}$ was reached and maintained for more than 70 days. An array of 8 crystals, called mini-tower, was used to check bolometers operation, readout electronics and DAQ. Results will be presented in terms of cooling power, electronic noise, energy resolution and preliminary background measurements.

XVII International Workshop on Neutrino Telescopes

13-17 March 2017

Venezia, Italy

${ }^{*}$ Speaker. 


\section{The CUORE experiment}

CUORE (Cryogenic Underground Observatory for Rare Events) aims to reveal the neutrinoless double decay $(0 v \beta \beta)$, if exists, of ${ }^{130} \mathrm{Te} .0 v \beta \beta$ is an hypothetical decay in which a nucleus changes its atomic number by two units, emitting two beta particles and no neutrinos [1]. The signature is the emmission of two beta particles whose total energy corresponds to the Q-value of the decay $\left(\mathrm{Q}_{\beta \beta}=2527 \mathrm{keV}\right.$ for $\left.{ }^{130} \mathrm{Te}\right)$. Its observation would confirm that neutrinos are Majorana particles and that the lepton number can be globally violated, moreover it would give us important indications regarding the neutrino mass scale and theyr mass hierarchy. CUORE started the data taking at the beginning of 2017 , and its data, combined with the predecessors Cuoricino and CUORE-0 [2], provided the most stringent lower limit of $0 v \beta \beta$ half-life.

The sensitivity of a double beta decay experiment depends on the number of potential decaying nuclei, the background index in the region of interest, the energy resolution and the experiment duration. For this reasons CUORE has been designed to contain more than $200 \mathrm{~kg}$ of isotope, and a data taking of 5 effective years has been scheduled. The low background has been achieved by placing the experiment underground, at Laboratori Nazionali del Gran Sasso, by surrounding the detector with passive lead shields and by selecting radiopure materials for the detectors. The target values are 0.01 counts $/ \mathrm{yr} / \mathrm{keV} / \mathrm{kg}$ in the region of interest for the background index and the energy resolution of $5 \mathrm{keV} \mathrm{FWHM} \mathrm{at} 2615 \mathrm{keV}$. The last requirement drove the choice of the bolometric technique as detection method. In CUORE the sensitive mass is composed by an array of 988 independent detectors operating at ultra-low temperature, called bolometers. The bolometers, arranged in 19 structures called towers, can be described as follows. The absorber is a single $\mathrm{TeO}_{2}$ crystal of $5 \times 5 \times 5 \mathrm{~cm}^{3}$, it is the sensitive volume in which the energy is deposited by the particle converted into heat. The thermistor is the sensor, thermally coupled to the absorber, used to measure the absorber temperature; in this case a Germanium semiconductor doped by neutron transmutation (Ge-NTD) is used. Finally the thermal link to a thermal bath restores the original bolometer temperature. In order to be sensitive enough, the bolometers must be operated at very low temperature; in particular the CUORE crystal are mantained at $\sim 15 \mathrm{mK}$ thanks to a custom cryostat that will be described in the next section.

\section{The CUORE cryostat}

The CUORE detector array is hosted in a custom dilution cryostat [3] (figure 1). Its peculiarity, apart from the extraordinary size, is that it is cryogen-free: in place of the liquid Helium five Pulse Tubes (PT) are used in the first phase of the cooling down. To reduce the injection of mechanical vibrations, the cryostat is suspendend to a structure called Main Support Plate (MSP), at the level of the second foor of the experimental hut. For the same reason the detector array is not rigidly coupled to the cryostat but it hungs from a structure (Y-Beam), connected to the MSP by means of three damping suspensions.

The cryostat can be divided in two, vacuum tight, volumes: the innermost is called Inner Vacuum Chamber (IVC) and it is surrounded by the external volume called Outer Vacuum Chamber (OVC). More precisely the cryostat can be divided into six volumes corresponding to different thermal stages, each one composed of a copper vessel and a surrounding plate. The names of the 


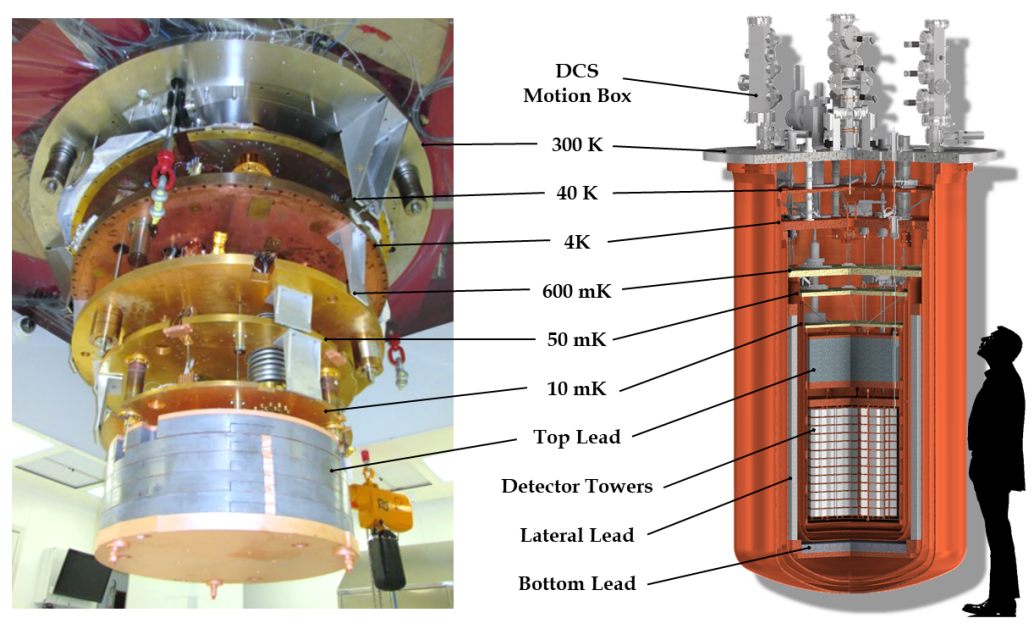

Figure 1: The CUORE cryostat.

vessels corresponds to their working temperature: $300 \mathrm{~K}, 40 \mathrm{~K}, 4 \mathrm{~K}, 600 \mathrm{mK}, 50 \mathrm{mK}$ and $10 \mathrm{mK}$. The $300 \mathrm{~K}$ plate, fixed to the MSP, hosts several elements such as the ports for the readout and thermometry cabling and the motion boxes of the Detector Calibration System (DCS). Inside the cryostat the cold fingers of the five pulse tubes are fixed to the $4 \mathrm{~K}$ plate, while the mixing chamber of the dilution unit is placed on the $10 \mathrm{mK}$ plate. Inside the $10 \mathrm{mK}$ vessel the detector array is protected by the top lead shield. In order to provide an analogue shielding in the other directions, a lateral and a bottom lead shields are also present, between the $600 \mathrm{mK}$ and the $4 \mathrm{~K}$ vessels. In particular these last two are made with the lead found in an ancent roman ship, sunken about 2000 years ago $\left({ }^{210} \mathrm{~Pb}<0.004 \mathrm{~Bq} / \mathrm{kg}\right)$. Furthermore, an external shield of modern lead can be raised up, from the ground floor to the cryostat level, during the operations. The Tower Support Plate (TSP), used to suspend the detector array, is located under the $10 \mathrm{mK}$ plate, just below the top lead shield.

\section{The commissioning runs}

After some preliminary tests of the Dilution Refrigerator and the Pulse Tubes, in the beginning of 2014, the cryostat was assembled in its final configuration and four test runs were performed, until the middle of 2016. Every run consisted in the integration of new parts in the apparatus followed by a cool down test; each one required several months. The cooling procedure is carried out in three steps. The first one is performed by the Fast Cooling System (FCS) which is used to flush gaseous Helium, at about $40 \mathrm{~K}$, inside the IVC. This technique permits to cool a mass of about 15 tonnes down to $50 \mathrm{~K}$ in about two weeks. When the temperature of $100 \mathrm{~K}$ is reached, five PTs are used to bring the apparatus down to $4 \mathrm{~K}$. Finally the base temperature of $10 \mathrm{mK}$ is reached thanks to a customized Dilution Refrigerator (DR), produced by Leiden Cryogenics in Netherlands, with a nominal cooling power of $5 \mu \mathrm{W}$ at $12 \mathrm{mK}$. The DR acts thanks to a mixture of ${ }^{3} \mathrm{He} /{ }^{4} \mathrm{He}$ that continuously flows through two condensing lines and reaches the mixing chamber. The condensing lines, they are two for redundancy, are termalized with two PTs in order to have the precooling, at $4 \mathrm{~K}$, of the incoming mixture. 
The goal of the first run was to characterize the DR and reach the base temperature below $10 \mathrm{mK}$. After an improvement of the thermal radiation shielding, required to insulate every stage from the radiation coming from warmer stages, the base temperature of $\simeq 6 \mathrm{mK}$ was obtained. The temperature measurements, in the $\mathrm{mK}$ range, were done with different thermometers: a Cerium Magnesium Nitrate $(\mathrm{CMN})$ magnetic thermometer, a Johnson noise thermometer and a fixed point thermometer. During the cool down, the dilution unit was able to bring the innermost part of the cryostat, corresponding to a thermal load of $440 \mathrm{~kg}$, from $3.8 \mathrm{~K}$ to $10 \mathrm{mK}$ in twelve hours. During this run a temperature oscillation, with a period of approximately 4 hours, was observed. It was visible only at low temperature, below $100 \mathrm{mK}$, and its amplitude was between 5 and $30 \mathrm{mK}$, depending on the cryostat configuration. Among the hypotheses, a possible explanation concerned mechanical vibrations induced by the PTs. Indeed the PT rotating motors and their compressors produce a significant vibration which was transmitted to the $300 \mathrm{~K}$, and then to the inner vessels, by the flex lines.

The main goals of the Test Run 2 were to acquire the first signals from real bolometers, have a first characterization of the detectors noise, measure the impact of the wiring on the thermalization and test the displacement procedure of the $\gamma$ sources. The CUORE wiring consists of 2600, 0.1 $\mathrm{mm}$ thick, wires which bring thermistor and heater signals from detectors to Front End electronics. Such a large temperature gradient (from $300 \mathrm{~K}$ to $10 \mathrm{mK}$ ) can cause a significant heat injection directly on the $10 \mathrm{mK}$ stage. For this reason the wires are connected to different thermalizations at $4 \mathrm{~K}, 600 \mathrm{mK}$ and $50 \mathrm{mK}$, in order to avoid the thermal power to be transmitted to the colder stage. In this phase the wiring was placed, checked for continuity, and it did not show an appreciable impact on the temperature of the $10 \mathrm{mK}$ stage: a base temperature of $7.3 \mathrm{mK}$ was reached. A leak on the dilution unit, in December of 2014, caused the interruption of the Test Run 2.

According to the initial plan, Run 3 should have been the last one but a delay in the production of the roman lead shields convinced the collaboration to proceed with the test by using the top lead only. This run highlighted the necessity of minor improvements on some subsystems. Moreover it provided new data about the temperature instability issue (i.e. the four hour long temperature oscillations) and definitely proved that they were due to mechanical inputs coming from the PTs. This effect has been reduced by taking some precautions concerning the cardanic joints inside the cryostat, the PTs and the machanical insulation of the cryostat.

A final run, the Test Run 4, was done to check cool down of the shielding. The lateral and bottom shields were added, so the thermal load in the $4 \mathrm{~K}$ stage reached the impressive mass of $\sim 14$ tonnes. Despite the large cryostat mass, the Fast Cooling System and PTs took sixteen days to cool the cryostat down to $4 \mathrm{~K}$. The base temperature of $(6.3 \pm 0.2) \mathrm{mK}$ was reached and the cryostat was mantained cold, in stable conditions, for about two months. Test Run 4 officially concluded the cryostat commissioning phase. After that moment, March 2016, the installation of the other subsystems - Faraday cage, electronics, DAQ - was done, followed by the complex phase of the detector installation that took one month. CUORE Data taking started in the beginning of 2017 and is currently going on.

\section{The Mini-tower run results}

The bolometer functionality has been tested starting from the Test Run 2 by means of an array 
of 8 crystals, called Mini-Tower. Differently from the CUORE detector towers, the Mini-Tower was first suspended from the $10 \mathrm{mK}$ plate, and not to the TSP; therefore larger noise, induced by the cryostat vibrations, was expected. Nevertheless, it gave the opportunity to check several important aspects of the commissioning and, after the insertion of the top lead during Run 3, the Mini-Tower was moved under the TSP.

During the Run 4, when the cryostat reached its final configuration, two Thorium sources were used to calibrate the eight Mini-Tower bolometers and to measure their energy resolution (figure 2). After several improvements, consisting in a reduction of the mechanical vibration and the optimization of the read-out electronics, two channels showed an energy resolution very close to the CUORE goal of $5 \mathrm{keV}$ (FWHM) at $2615 \mathrm{keV}$. More in general the resolution obtained by the combined specturm from the five best cahannels was $10 \mathrm{keV}$ (FWHM at $2615 \mathrm{keV}$ ). Finally the external lead shield was lifted up to obtain a configuration as close as possible to the final one. The acquired specrum did not show evidence of unaccounted background sources and this has been confirmed by the first CUORE data in which the rates are consistent with the background model, leaving unchanged the predicted sensitivity [4].

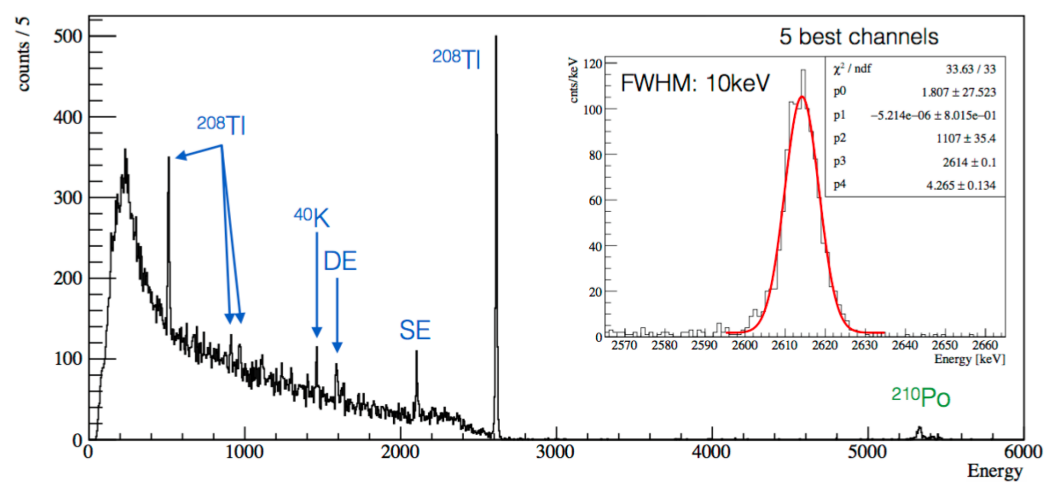

Figure 2: Outer: background energy spectrum acquired with the Mini-Tower. Inner: energy resolution of the five best channel measured with the ${ }^{208} \mathrm{Tl}$ peak.

\section{References}

[1] M. Goeppert-Mayer, "Double beta-disintegration," Phys. Rev. 48 (1935) 512. doi:10.1103/PhysRev.48.512

[2] K. Alfonso et al. [CUORE Collaboration], "Search for Neutrinoless Double-Beta Decay of ${ }^{130} \mathrm{Te}$ with CUORE-0,” Phys. Rev. Lett. 115 (2015) no.10, 102502 doi:10.1103/PhysRevLett.115.102502 [arXiv:1504.02454 [nucl-ex]].

[3] C. Ligi et al. [CUORE Collaboration], "The CUORE Cryostat: A 1-Ton Scale Setup for Bolometric Detectors," J. Low. Temp. Phys. 184 (2016) no.3-4, 590 doi:10.1007/s10909-015-1389-4 [arXiv:1603.03306 [physics.ins-det]].

[4] C. Alduino et al. [CUORE Collaboration], "The projected background for the CUORE experiment," Eur. Phys. J. C 77 (2017) no.8, 543 doi:10.1140/epjc/s10052-017-5080-6 [arXiv:1704.08970 [physics.ins-det]]. 